\title{
Partial nephrectomy provides equivalent oncologic outcomes and better renal function preservation than radical nephrectomy for pathological T3a renal cell carcinoma: A meta-analysis
}

Huan Deng 1, 2, 3, Yan Fan 2, 3, Feifei Yuan 2, 3, Li Wang 2, 3, Zhengdong Hong 2, Jinfeng Zhan ${ }^{4}$, Wenxiong Zhang ${ }^{1}$

${ }^{1}$ Department of Thoracic Surgery, The Second Affiliated Hospital of Nanchang University, Nanchang, China; ${ }^{2}$ Department of Urology, The Second Affiliated Hospital of Nanchang University, Nanchang, China; ${ }^{3}$ Jiangxi Medical College, Nanchang University, Nanchang, China $;{ }^{4}$ Department of Nephrology, The Second Affiliated Hospital of Nanchang University, Nanchang, China

\section{ABSTRACT}

Purpose: Radical nephrectomy $(\mathrm{RN})$ is the standard surgical type for pathological stage T3a (pT3a) renal cell carcinoma (RCC). Recently, some studies have suggested equivalence between partial nephrectomy (PN) and RN for oncologic control and have shown the benefits of PN for better renal function. We conducted this meta-analysis to assess oncologic outcomes, perioperative outcomes and renal function between two groups among patients with pT3a RCC.

Materials and methods: PubMed, Scopus, Web of Science, Science Direct, Ovid MEDLINE, The Cochrane Library, Embase and Google Scholar were searched for eligible articles. The endpoints of the final analysis included overall survival (OS), cancer-specific survival (CSS), recurrence-free survival (RFS), surgical complications, operative time, estimated blood loss (EBL), serum creatinine and estimated glomerular filtration rate (eGFR).

Results: Twelve studies of moderate to high quality, including 14.152 patients, were examined. PN showed superiority for renal functional preservation, providing higher eGFR (WMD=12.48mL/min; 95\%CI: 10.28 to 14.67 ; $\mathrm{P}<0.00001$ ) and lower serum creatinine $(\mathrm{WMD}=-0.31 \mathrm{mg} / \mathrm{dL} ; 95 \% \mathrm{CI}$ : -0.40 to $-0.21 ; \mathrm{P}<0.00001)$. There were no significant differences between $\mathrm{PN}$ and RN regarding operative time, EBL, surgical complications, OS, RFS and CSS. Despite inherent selection bias, most pooled estimates were consistent in sensitivity analysis and subgroup analysis. More positive margins were found in the PN group ( $\mathrm{RR}=2.42 ; 95 \% \mathrm{CI}$ : $1.25-4.68 ; \mathrm{P}=0.009$ ).

Conclusions: PN may be more suitable for treating pT3a RCC than RN because it provides a similar survival time (OS or RFS) and superior renal function. Nevertheless, this result is still disputed, and more high-quality studies are required.

\section{ARTICLE INFO}

Wenxiong Zhang

http://orcid.org/0000-0003-2962-0847

\section{Keywords:}

Nephrectomy; Carcinoma,

Renal Cell; Meta-Analysis

[Publication Type]

Int Braz J Urol. 2021; 47: 46-60

Submitted for publication:

March 04, 2020

Accepted after revision:

March 09, 2020

Published as Ahead of Print:

April 3, 2020 


\section{INTRODUCTION}

Renal cell carcinoma (RCC) is the eighth most common type of cancer in the United States, with an incidence of 65.340, and caused 14.970 deaths in 2018 (1). Local RCC is the most common manifestation, and nearly one-third of patients are diagnosed with T3-T4 RCC (2). Recently, the oncologic outcomes of partial nephrectomy (PN) were found to have oncologic results similar to those of radical nephrectomy (RN) (3).

$\mathrm{PN}$ is recommended by the European Association of Urology (EAU) and National Comprehensive Cancer Network (NCCN) guidelines as the standard choice for T1a-b RCC (4). Additionally, some articles have shown that PN for T2 or greater renal tumors may offer oncologic outcomes similar to those of RN (5). The most attractive and beneficial feature of PN compared with RN is better renal function (6), which might decrease the risk of cardiovascular and metabolic events that may ultimately translate into better overall survival (OS) (7). However, the only randomized control trial (RCT) EORTC 30904 failed to show significant advantages that favored PN in these terms, despite showing oncologic similarity (3). Therefore, it remains controversial whether $\mathrm{PN}$ is a feasible choice for pathological T3a (pT3a) RCC.

To resolve this controversy, this article systematically evaluated and analyzed the therapeutic efficacy of PN and RN among patients with pT3a RCC to evaluate OS, cancer-specific survival (CSS), recurrence-free survival (RFS), surgical complications, perioperative outcomes and renal functions between PN and RN to provide evidence-based data for patients with pT3a RCC with regard to the selection of surgical procedures.

\section{MATERIALS AND METHODS}

Our meta-analysis was performed in accordance with Preferred Reporting Items for Systematic Review and Meta-Analysis (PRISMA) guidelines (Registration information: CRD42020153787).

\section{Search strategy \\ PubMed, Scopus, Web of Science, Scien-} ce Direct, Ovid MEDLINE, The Cochrane Library,
Embase and Google Scholar were searched up to April 15, 2019, to identify relevant articles comparing $\mathrm{PN}$ to $\mathrm{RN}$ for $\mathrm{pT} 3 \mathrm{a} \mathrm{RCC}$. The following terms were used: "renal cell carcinoma", "pathological T3a", "partial nephrectomy" and "radical nephrectomy". We also searched the references of included studies to find further eligible studies.

\section{Inclusion criteria}

Studies that satisfied the following criteria were included: 1) patients diagnosed with pT3a RCC; 2) comparison of PN with RN; 3) final outcomes of RFS, OS, CSS, surgical complications, estimated blood loss (EBL), operative time, serum creatinine and estimated glomerular filtration rate (eGFR). We excluded reviews lacking raw data, meta-analyses, conference abstracts, animal experiments and articles with repeated data.

\section{Data extraction}

Two investigators abstracted the following information independently: year of publication, first author, study origin, study period, study design, number of participants, participant characteristics (age, sex, tumor size, pathological type, surgical approach and so on), oncologic outcomes (OS, RFS, CSS), perioperative outcomes (EBL, operative time, positive margins), surgical complications (intraoperative and postoperative complications) and renal function (eGFR, serum creatinine). A third investigator settled differences in all situations.

We used the multivariable adjusted hazard ratio (HR), which takes into consideration the quantity and time of events instead of OR, to analyze oncologic outcomes. HRs and 95\%CIs were obtained directly if Cox multivariate survival analysis was conducted; otherwise, HRs and 95\%CIs were extracted from Kaplan-Meier curves according to Tierney et al. $(8,9)$. Some 3-year all-cause mortality, 5-year all-cause mortality, 3-year recurrence rate, 5-year recurrence rate, 2-year cancer-specific mortality (CSS) and 5-year CSS data were also extracted from survival curves because of the lack of available data in the included articles.

\section{Quality assessment}

The quality of each study was assessed using the Newcastle-Ottawa Scale (NOS) for retrospective 
studies, which includes questions on three major projects: selection, comparability and exposure. A total score of 8-9 points was considered high-quality; 6-7 was considered medium-quality (10).

\section{Statistical analysis}

This meta-analysis was performed using Review Manager (version 5.2, The Nordic Cochrane Centre) and STATA (version 12.0, Stata Corp). Risk ratios (RR) with 95\% confidence intervals (CIs) were used to analyze 3-year all-cause mortality, 5-year all-cause mortality, 3-year recurrence rate, 5-year recurrence rate, 2-year CSS, 5-year CSS and positive margins (RR $>1$ supports $\mathrm{PN}$; RR $<1$ supports RN). Hazard ratios (HR) with 95\%CIs were used to analyze OS, RFS and CSS (HR >1 supports RN; HR $<1$ supports PN). Weighted mean difference (WMD) and 95\%CIs were employed to assess operative time, EBL, eGFR and serum creati- nine. Subgroup analysis of HR of OS, RFS and CSS were performed to determine whether the results would vary according to upstaging, adjustment/ matching, study center, tumor size and follow-up time. Heterogeneity was examined using the $\chi^{2}$ test and $\mathrm{I}^{2}$ statistic. If $\mathrm{I}^{2}>50 \%$ or $\mathrm{P}<0.1$ for the $\chi^{2}$ test, reflecting significant heterogeneity, the random-effects model was used; if not, the fixed-effects model was used. To enhance robustness, sensitivity analysis was performed to determine the effects of variables. Publication bias was evaluated using Begg's test and Egger's test. $\mathrm{P}<0.05$ indicated statistical significance.

\section{RESULTS}

Search results and study quality assessment

Figure-1 shows the process of study selection. Ultimately, 12 studies including 14.152 patients (2486 PN and 11.666 RN) were selected

Figure 1 - Flow chart of study selection.
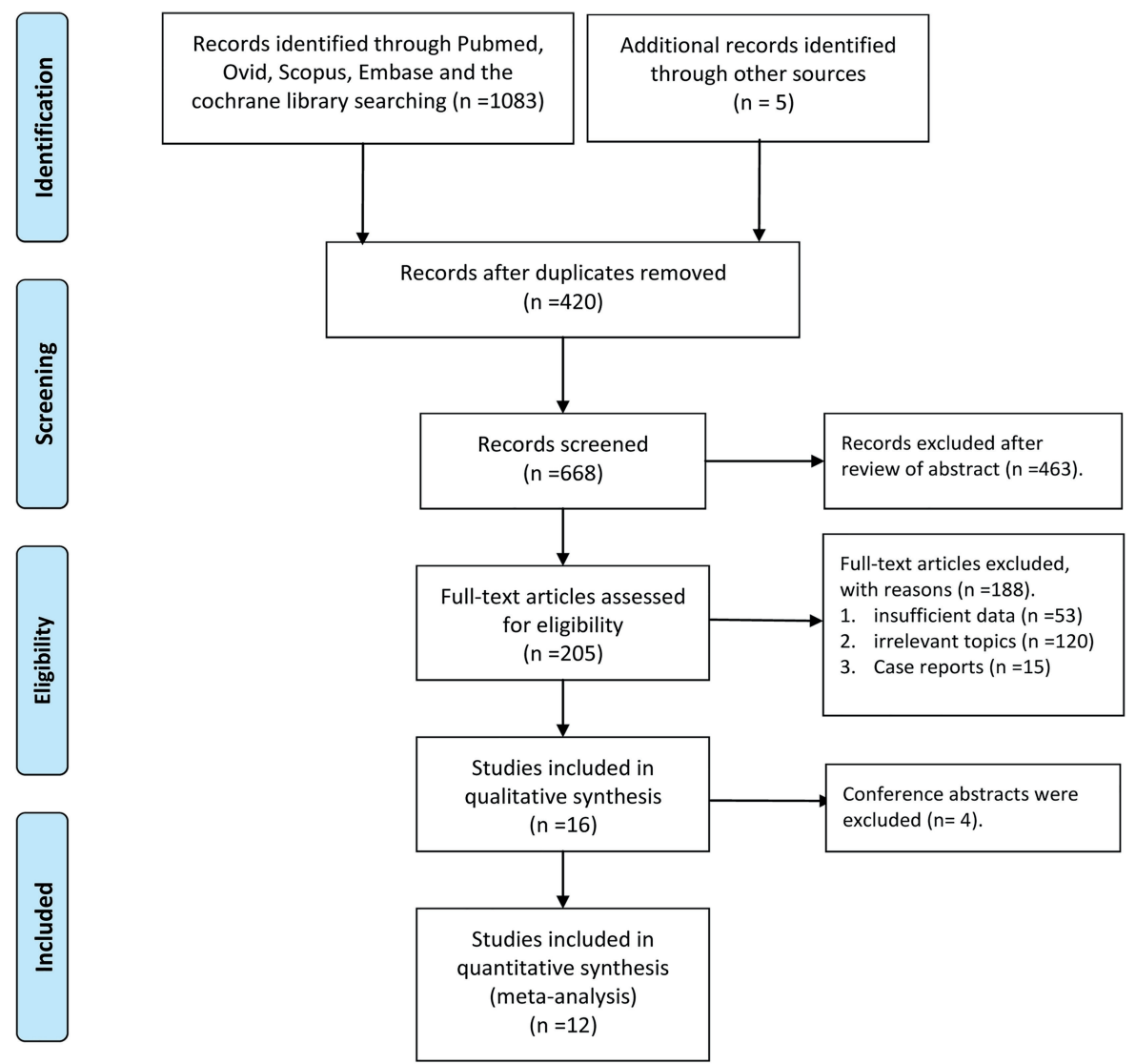
for this meta-analysis (11-22). Of the 12 studies, four were high quality and eight medium quality (Table-S1). Table-1 provides the baseline characteristics and major evaluation indices of the included articles.

\section{Oncologic outcomes}

We assessed oncologic outcomes between PN and RN groups based on OS, RFS, and CSS.

Four studies compared the HR of OS (heterogeneity: $\left.\mathrm{P}=1.00, \mathrm{I}^{2}=0 \%\right)$. No significant difference was found between $\mathrm{PN}$ and $\mathrm{RN}(\mathrm{HR}=0.92,95 \% \mathrm{CI}$ : 0.26-3.30, $\mathrm{P}=0.89$; Figure-2A).
Eight studies compared the HR of RFS (heterogeneity: $\mathrm{P}=0.98, \mathrm{I}^{2}=0 \%$ ). No significant difference was found (HR=1.26, 95\%CI: 0.70-2.29, $\mathrm{P}=0.44$; Figure-2B).

Five studies compared the HR of CSS (heterogeneity: $\left.\mathrm{P}=1.00, \mathrm{I}^{2}=0 \%\right)$. No significant difference was found (HR=1.01, 95\%CI: 0.64-1.58, $\mathrm{P}=0.98$; Figure-2C).

Five studies compared total CSS (heterogeneity: $\mathrm{P}=0.13, \mathrm{I}^{2}=43 \%$ ). No significant difference was found between $\mathrm{PN}$ and $\mathrm{RN}(\mathrm{RR}=0.91,95 \% \mathrm{CI}$ : 0.47-1.74, $\mathrm{P}=0.77$; Figure-3A). Moreover, there

Table S1 - Quality assessment of all included studies according to Newcastle-Ottawa Scale.

\begin{tabular}{|c|c|c|c|c|c|c|c|c|c|}
\hline \multirow[b]{2}{*}{ Study } & \multicolumn{4}{|c|}{ Selection } & \multirow{2}{*}{$\begin{array}{c}\text { Comparability } \\
\text { Comparability } \\
\text { of cases and } \\
\text { controls on } \\
\text { the basis of } \\
\text { the design or } \\
\text { analysis }\end{array}$} & \multicolumn{3}{|c|}{ Exposure } & \multirow[b]{2}{*}{$\begin{array}{l}\text { Quality } \\
\text { score }\end{array}$} \\
\hline & $\begin{array}{c}\text { Is the } \\
\text { case } \\
\text { definition } \\
\text { adequate? }\end{array}$ & $\begin{array}{l}\text { Representativeness } \\
\text { of the cases }\end{array}$ & $\begin{array}{l}\text { Selection } \\
\text { of } \\
\text { Controls }\end{array}$ & $\begin{array}{l}\text { Definition } \\
\text { of } \\
\text { Controls }\end{array}$ & & $\begin{array}{l}\text { Ascertainment } \\
\text { of exposure }\end{array}$ & $\begin{array}{c}\text { Same } \\
\text { method of } \\
\text { ascertainment } \\
\text { for cases and } \\
\text { controls }\end{array}$ & $\begin{array}{l}\text { Non- } \\
\text { Response } \\
\text { rate }\end{array}$ & \\
\hline $\begin{array}{l}\text { Jeldres, et al. } \\
\text { (11) }\end{array}$ & $\star$ & $\star$ & & $\star$ & $\star \star$ & $\star$ & $\star$ & $\star$ & 8 \\
\hline $\begin{array}{l}\text { Hansen, et al. } \\
\text { (12) }\end{array}$ & $\star$ & $\star$ & & $\star$ & $\star \star$ & $\star$ & $\star$ & & 7 \\
\hline Polo et al. (13) & $\star$ & $\star$ & & $\star$ & $\star$ & $\star$ & $\star$ & $\star$ & 7 \\
\hline Oh et al. (14) & $\star$ & $\star$ & & $\star$ & $\star$ & $\star$ & $\star$ & $\star$ & 7 \\
\hline Jeong et al. (15) & $\star$ & $\star$ & & $\star$ & $\star$ & $\star$ & $\star$ & $\star$ & 7 \\
\hline Nayak et al. (16) & $\star$ & $\star$ & & $\star$ & $\star$ & $\star$ & $\star$ & $\star$ & 7 \\
\hline Shah et al. (17) & $\star$ & $\star$ & & $\star$ & $\star$ & $\star$ & $\star$ & $\star$ & 7 \\
\hline $\begin{array}{l}\text { Andrade et al. } \\
\text { (18) }\end{array}$ & $\star$ & $\star$ & & $\star$ & $\star \star$ & $\star$ & $\star$ & $\star$ & 8 \\
\hline Peng et al. (19) & $\star$ & $\star$ & & $\star$ & $\star \star$ & $\star$ & $\star$ & $\star$ & 8 \\
\hline Shvero et al. (20) & $\star$ & $\star$ & & $\star$ & $\star$ & $\star$ & $\star$ & $\star$ & 7 \\
\hline $\begin{array}{l}\text { Srivastava et al. } \\
\text { (21) }\end{array}$ & $\star$ & $\star$ & & $\star$ & $\star \star$ & $\star$ & $\star$ & $\star$ & 8 \\
\hline Lee et al. (22) & $\star$ & $\star$ & & $\star$ & $\star$ & $\star$ & $\star$ & $\star$ & 7 \\
\hline
\end{tabular}


Table 1 - Characteristics of the included studies.

\begin{tabular}{|c|c|c|c|c|c|c|c|c|c|c|c|c|c|}
\hline Study & & $\begin{array}{l}\text { Study } \\
\text { period }\end{array}$ & $\begin{array}{l}\text { Study } \\
\text { design }\end{array}$ & $\begin{array}{l}\text { Study } \\
\text { Origin }\end{array}$ & Groups & $\begin{array}{l}\text { patients } \\
\text { (n) }\end{array}$ & Tumor size(cm) & $\operatorname{ccRCC}(\mathrm{n})$ & $\begin{array}{c}\text { Fuhrman } \\
\text { grade III/ } \\
\text { IV(n) }\end{array}$ & $\begin{array}{l}\text { Surgical } \\
\text { approach }\end{array}$ & $\begin{array}{l}\text { Adjustment/ } \\
\text { matching }\end{array}$ & $\begin{array}{c}\text { FU } \\
\text { (month) }\end{array}$ & SQ \\
\hline $\begin{array}{l}\text { Jeldres et al, } \\
\text { (11) }\end{array}$ & 2009 & $\begin{array}{l}1984- \\
2001\end{array}$ & $\begin{array}{l}\text { RTP, } \\
\text { MI }\end{array}$ & $\begin{array}{c}\text { Canada, } \\
\text { Italy, } \\
\text { France }\end{array}$ & $\begin{array}{l}\text { PN vs. } \\
\text { RN }\end{array}$ & $30 / 63$ & $1.5-9.5 / 1.5-10.5$ & $27 / 58$ & $12 / 32$ & NS & Yes & 50.4 & 8 \\
\hline $\begin{array}{l}\text { Hansen et al, } \\
\text { (12) }\end{array}$ & 2012 & $\begin{array}{l}1988- \\
2008\end{array}$ & $\begin{array}{l}\text { RTP, } \\
\text { MI }\end{array}$ & USA & $\begin{array}{l}\text { PN vs. } \\
\text { RN }\end{array}$ & $477 / 477$ & $2.4-4.5 / 2.5-4.8$ & $354 / 355$ & NS & NS & Yes & NS & 7 \\
\hline $\begin{array}{l}\text { Polo et al, } \\
\text { (13) }\end{array}$ & 2012 & $\begin{array}{l}1994- \\
2009\end{array}$ & $\begin{array}{l}\text { RTP, } \\
\text { NS }\end{array}$ & France & $\begin{array}{c}\text { PN vs. } \\
\text { RN }\end{array}$ & $10 / 33$ & $2.7 / 6.0$ & $5 / 25$ & $3 / 15$ & NS & No & 45 & 7 \\
\hline $\begin{array}{l}\text { Oh et al, } \\
\text { (14)a }\end{array}$ & 2014 & $\begin{array}{l}2000- \\
2010\end{array}$ & $\begin{array}{l}\text { RTP, } \\
\text { MI }\end{array}$ & Korea & $\begin{array}{l}\text { PN vs. } \\
\text { RN }\end{array}$ & $45 / 298$ & $3.50 \pm 1.55 / 7.99 \pm 3.68$ & $36 / 247$ & $23 / 211$ & $\begin{array}{c}\text { Open/Lap/ } \\
\text { Rob }\end{array}$ & No & 43 & 7 \\
\hline $\begin{array}{l}\text { Jeong et al, } \\
\text { (15) }\end{array}$ & 2016 & $\begin{array}{l}2001- \\
2013\end{array}$ & $\begin{array}{l}\text { RTP, } \\
\text { SC }\end{array}$ & Korea & $\begin{array}{l}\text { PN vs. } \\
\text { RN }\end{array}$ & $37 / 54$ & NS & NS & $58 \mathrm{c}$ & $\begin{array}{c}\text { Open/Lap/ } \\
\text { Rob }\end{array}$ & No & 50.8 & 7 \\
\hline $\begin{array}{l}\text { Nayak et al, } \\
\text { (16) }\end{array}$ & 2016 & $\begin{array}{l}2009- \\
2015\end{array}$ & PRO & Canada & $\begin{array}{l}\text { PN vs. } \\
\text { RN }\end{array}$ & $66 / 68$ & $3.5-5.7$ & NS & $76 \mathrm{c}$ & Open/MIS & No & 23 & 7 \\
\hline $\begin{array}{l}\text { Shah et al, } \\
\text { (17) }\end{array}$ & 2017 & $\begin{array}{l}2006- \\
2014\end{array}$ & $\begin{array}{l}\text { RTP, } \\
\text { SC }\end{array}$ & USA & $\begin{array}{l}\text { PN vs. } \\
\text { RN }\end{array}$ & $49 / 91$ & $4.2 / 5.5$ & $41 / 86$ & NS & Lap/open & No & 38 & 7 \\
\hline $\begin{array}{l}\text { Andrade et } \\
\text { al, (18) }\end{array}$ & 2017 & $\begin{array}{l}2005- \\
2015\end{array}$ & $\begin{array}{l}\text { RTP, } \\
\text { SC }\end{array}$ & USA & $\begin{array}{l}\text { PN vs. } \\
\text { RN }\end{array}$ & $70 / 70$ & $3.0-5.2 / 3.9-5.4$ & $50 / 64$ & $43 / 40$ & Rob & Yes & 20 & 8 \\
\hline $\begin{array}{l}\text { Peng et al, } \\
\text { (19) }\end{array}$ & 2017 & $\begin{array}{l}2007- \\
2012\end{array}$ & $\begin{array}{l}\text { RTP, } \\
\text { SC }\end{array}$ & China & $\begin{array}{l}\text { PN vs. } \\
\text { RN }\end{array}$ & $18 / 18$ & $5.27 \pm 1.50 / 5.03 \pm 1.42$ & $13 / 13$ & $6 / 6$ & Open/Lap & Yes & 35.5 & 8 \\
\hline $\begin{array}{l}\text { Shvero et al, } \\
\text { (20) }\end{array}$ & 2018 & $\begin{array}{l}1987- \\
2015\end{array}$ & $\begin{array}{l}\text { RTP, } \\
\text { MI }\end{array}$ & Israel & $\begin{array}{c}\text { PN vs. } \\
\text { RN }\end{array}$ & $48 / 86$ & $2.8-5.2 / 5-9.5$ & $41 / 67$ & $25 / 53$ & NS & No & $55.2 / 48.8$ & 7 \\
\hline $\begin{array}{l}\text { Srivastava et } \\
\text { al, (21) b }\end{array}$ & 2018 & $\begin{array}{l}1998- \\
2013\end{array}$ & $\begin{array}{l}\text { RTP, } \\
\text { MI }\end{array}$ & USA & $\begin{array}{c}\text { PN vs. } \\
\text { RN }\end{array}$ & $1579 / 10250$ & $2.5-5.0 / 4.9-9.0$ & $791 / 5997$ & $541 / 4482$ & NS & No & $36 / 37$ & 8 \\
\hline $\begin{array}{l}\text { Lee et al, } \\
\text { (22) }\end{array}$ & 2018 & $\begin{array}{l}1997- \\
2016\end{array}$ & $\begin{array}{l}\text { RTP, } \\
\text { SC }\end{array}$ & Korea & $\begin{array}{c}\text { PN vs. } \\
\text { RN }\end{array}$ & $57 / 158$ & $3.7-6.2$ & $175 \mathrm{c}$ & $145 \mathrm{c}$ & LAP & No & 39 & 7 \\
\hline
\end{tabular}

$\mathbf{R T P}=$ retrospective; $\mathbf{P R O}=$ prospective; $\mathbf{M I}$ = multi-institutional; $\mathbf{S C}=$ single center; FU = Follow-up; $\mathbf{L a p}=$ laparoscopic; $\mathbf{R o b}=$ robotic; $\mathbf{c c R C C}=$ clear-cell renal cell carcinoma; $\mathbf{M I S}=$ minimally invasive surgery; $\mathbf{N S}$ = not specified; $\mathbf{S} \mathbf{Q}=$ study quality according to the Newcastle-Ottawa scale

$a=$ The group reported two separate subgroup analyses for the same data set.

${ }^{b}=$ The group reported three separate subgroup analyses for the same data set.

${ }^{c}=$ These studies only provide overall numbers, without providing numbers of PN and RN groups respectively.

were no significant differences regarding 2-year CSS (RR=0.73, 95\%CI: 0.43-1.22, $\mathrm{P}=0.23$; Figure$-3 \mathrm{~B})$ and 5-year CSS (RR=0.92, 95\%CI: 0.66-1.27, $\mathrm{P}=0.60$; Figure- $3 \mathrm{C}$ ).

There was no significant difference between the two groups regarding 3-year all-cause mortality $(\mathrm{RR}=0.58,95 \% \mathrm{CI}$ : 0.31-1.10, $\mathrm{P}=0.10$; Figure-4A) or 5-year all-cause mortality $(\mathrm{RR}=0.64,95 \% \mathrm{CI}$ : 0.24-1.73, $\mathrm{P}=0.38$; Figure-4B).

Furthermore, no significant difference was found between the two groups for the 3-year recurrence rate ( $R R=0.88,95 \% C I$ : 0.48-1.60, $\mathrm{P}=0.67$; Figure-5A) or the 5 -year recurrence rate $(R R=0.67$, 95\%CI: 0.31-1.48, $\mathrm{P}=0.32$; Figure-5B). 
Figure 2 - Forest plots of HR of OS (a), RFS (b) and CSS (c) associated with PN versus RN.

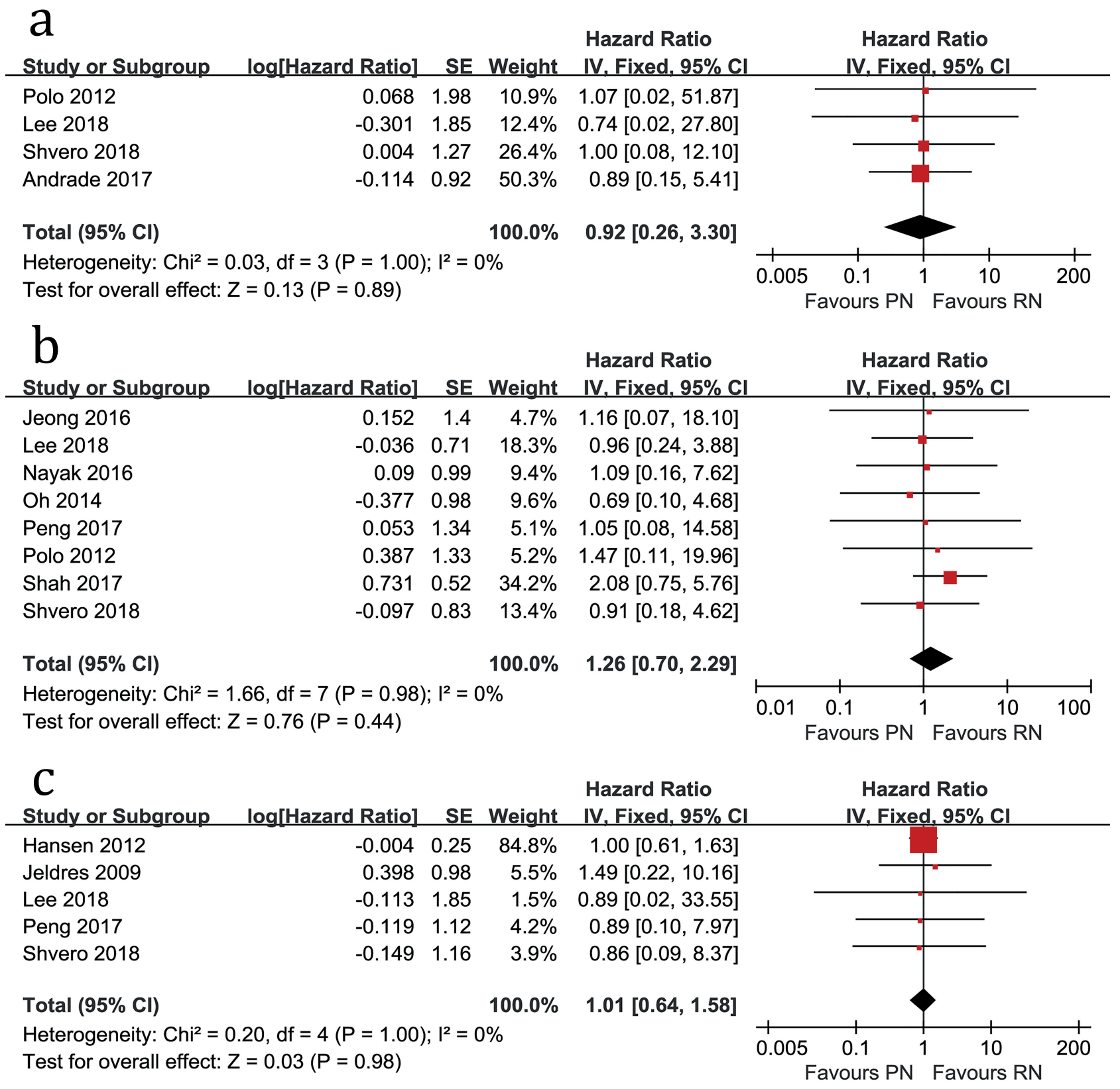

Surgical complications

Only one included study (Oh, 2014) reported intraoperative and postoperative complications, with no significant differences regarding intraoperative complications (15.6\% vs. $14.4 \%, \mathrm{P}=0.842$ ) or postoperative complications (13.3\% vs. $12.4 \%, \mathrm{P}=0.844)$.
Furthermore, no significant differences were found between the two groups regarding prolonged bleeding (2.2\% vs. $4.4 \%, \mathrm{P}=0.499)$; wound problems $(2.2 \%$ vs. $1.7 \%, \mathrm{P}=0.795)$; urine leakage ( $0 \%$ vs. $0.3 \%, P=0.697$ ); prolonged ileus $(2.2 \%$ vs. $2.7 \%, P=0.856)$ and others $(6.7 \%$ vs. $3.4 \%, \mathrm{P}=0.278)(14)$. 
Perioperative outcomes

Two studies compared EBL (heterogeneity: $\left.\mathrm{P}=0.11, \mathrm{I}^{2}=61 \%\right)$. No significant difference was found (WMD $=-177.67 \mathrm{~mL}$; 95\% $\mathrm{CI}$ : $-467.78 \mathrm{~mL}$ to 112.44mL; $\mathrm{P}=0.23$; Figure-6A).

Two studies compared operative time (heterogeneity: $\left.\mathrm{P}=0.50, \mathrm{I}^{2}=0 \%\right)$. No significant difference was found $(\mathrm{WMD}=-16.99 \mathrm{~min} ; 95 \% \mathrm{CI}$ : $-34.35 \mathrm{~min}$ to $0.38 \mathrm{~min} ; \mathrm{P}=0.06$; Figure-6B).
Six studies compared positive margins (heterogeneity: $\mathrm{P}=0.25 \mathrm{I}^{2}=25 \%$ ), and $\mathrm{PN}$ exhibited a higher incidence $(\mathrm{RR}=2.42$; 95\%CI: 1.25-4.68; $\mathrm{P}=0.009$; Figure-6C).

Postoperative renal function

Three studies compared eGFR (heterogeneity: $\mathrm{P}=0.54, \mathrm{I} 2=0 \%$ ). $\mathrm{PN}$ had a higher eGFR compared with $\mathrm{RN}(\mathrm{WMD}=12.48 \mathrm{~mL} / \mathrm{min}$; 95\%CI:

Figure 3 - Forest plots of total CSS (a), 2-year-CSS (b) and 5-year CSS(c) associated with PN versus RN.

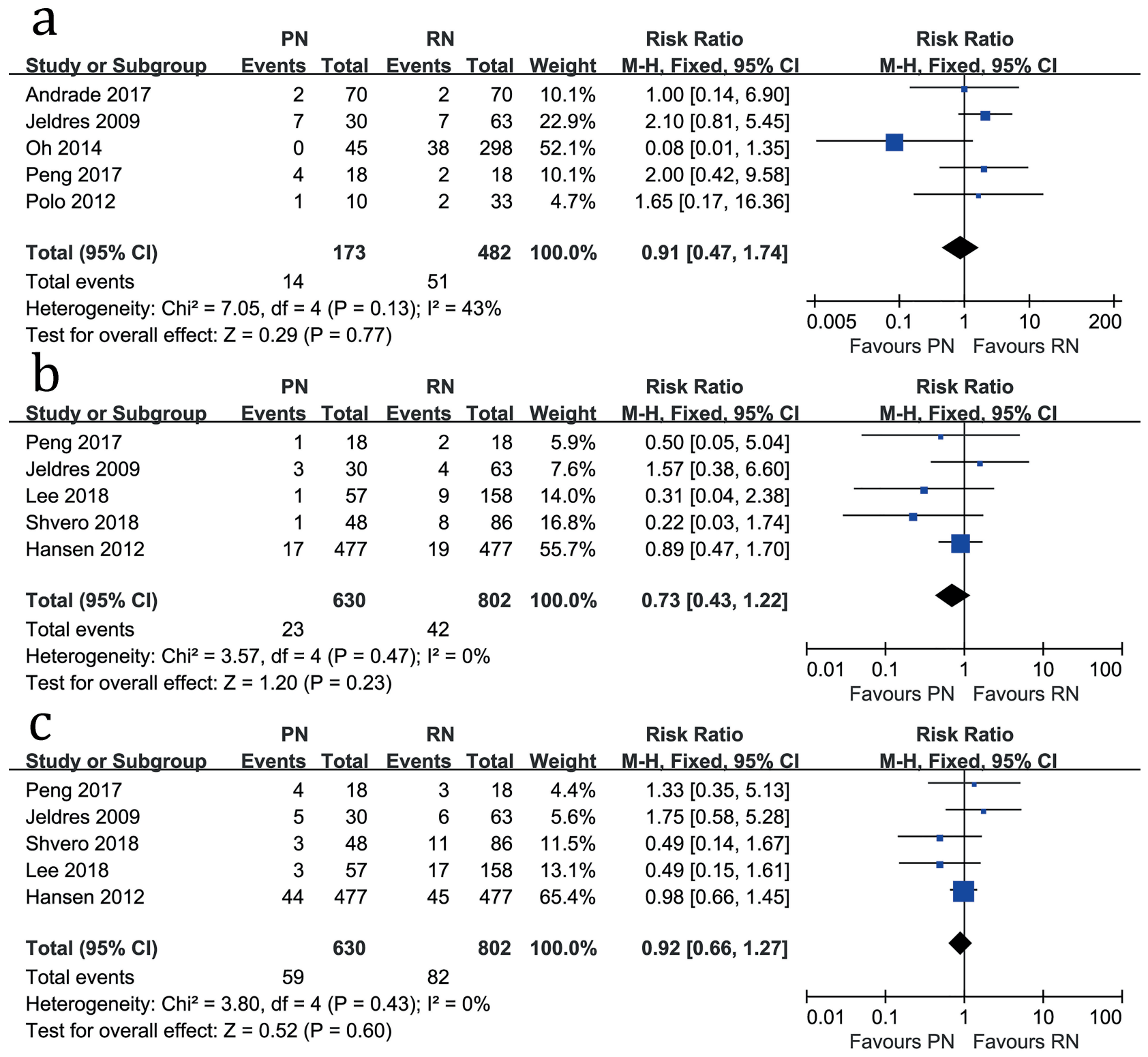


Figure 4 - Forest plots of 3-year all-cause mortality (a) and 5-year all-cause mortality (b) associated with PN versus RN.

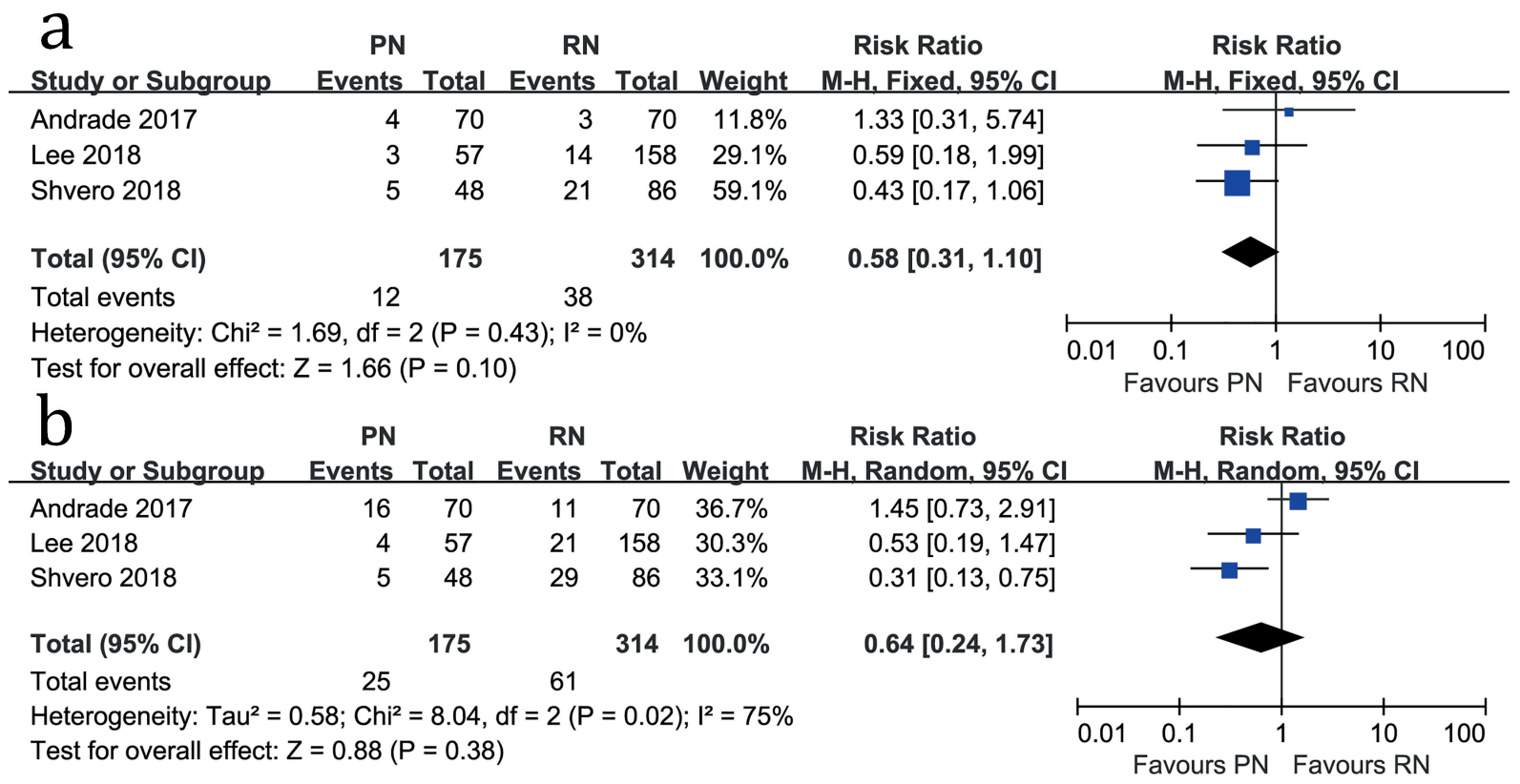

Figure 5 - Forest plots of 3-year recurrence rate (a) and 5-year recurrence rate (b) associated with PN versus RN.

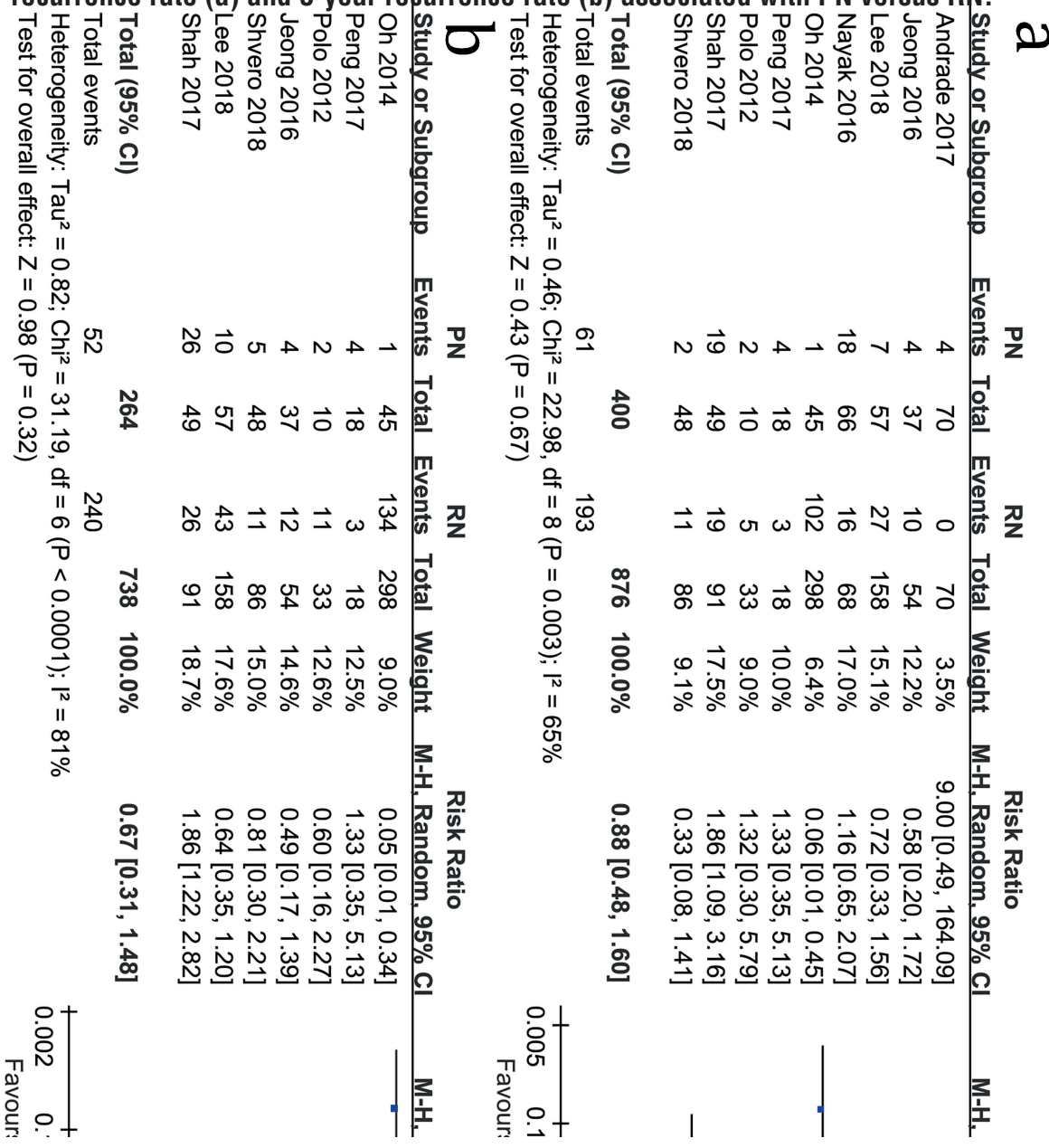


Figure 6 - Forest plots of EBL (a), operative time (b) and positive margins (c) associated with PN versus RN.

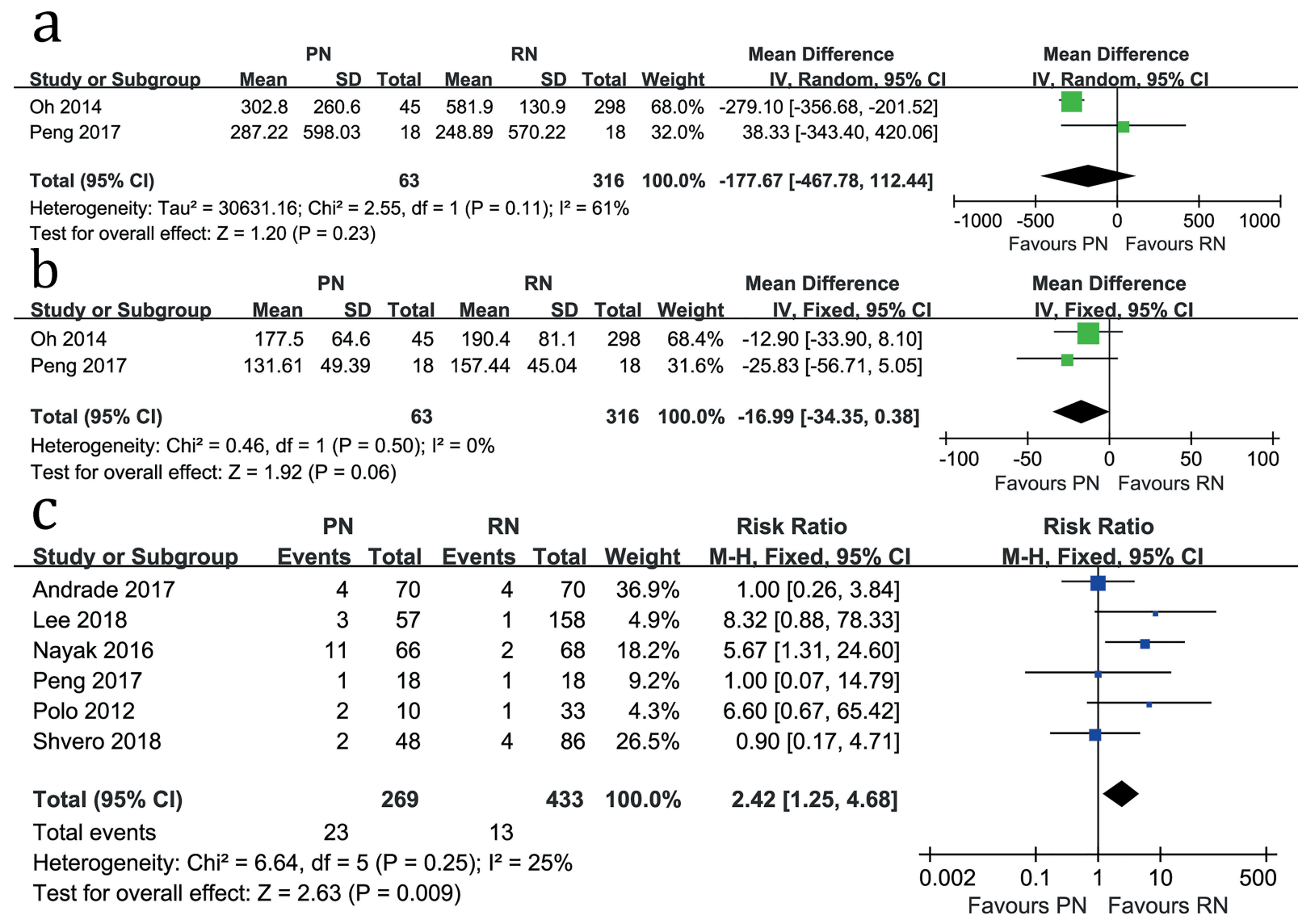

$10.28 \mathrm{~mL} / \mathrm{min}$ to $14.67 \mathrm{~mL} / \mathrm{min} ; \mathrm{P}<0.00001$; $\mathrm{Fi}-$ gure-7A).

Two studies compared serum creatinine (heterogeneity: $\mathrm{P}=0.91, \mathrm{I}^{2}=0 \%$ ), with $\mathrm{RN}$ being associated with higher levels compared with $\mathrm{PN}(\mathrm{WMD}=-0.31 \mathrm{mg} / \mathrm{dL} ; 95 \% \mathrm{CI}:-0.40 \mathrm{mg} / \mathrm{dL}$ to $-0.21 \mathrm{mg} / \mathrm{dL} ; \mathrm{P}<0.00001$; Figure-7B).

\section{Subgroup analysis}

To determine whether the oncologic outcomes of PN versus RN were robust across subgroups, pooled HRs of OS, RFS and CSS were estimated by upstaging, adjustment/matching, study center, tumor size and follow-up time. No statistically significant differences were found in any of the subgroup analyses of HR of OS, RFS and CSS between PN and RN (Table-2).
Sensitivity analysis

Based on sensitivity analysis, 2-year CSS, 5-year CSS, 3-year recurrence rate and 5-year recurrence rate, HR of OS, CSS and RFS were all robust, with consistent findings.

\section{Publication Bias}

Proof of publication bias was not found according to the HR of OS (Begg's test, $\mathrm{P}=1.000$; Egger's test, $\mathrm{P}=0.969$; Figure-S1A), RFS (Begg's test, $\mathrm{P}=0.711$; Egger's test, $\mathrm{P}=0.165$; Figure-S1B) and CSS (Begg's test, $\mathrm{P}=0.806$; Egger's test, $\mathrm{P}=0.900$; Figure-S1C).

\section{DISCUSSION}

This is the first meta-analysis of the oncologic outcomes, surgical complications, periope- 
Figure 7 - Forest plots of eGFR (a) and serum creatinine (b) associated with PN versus RN.

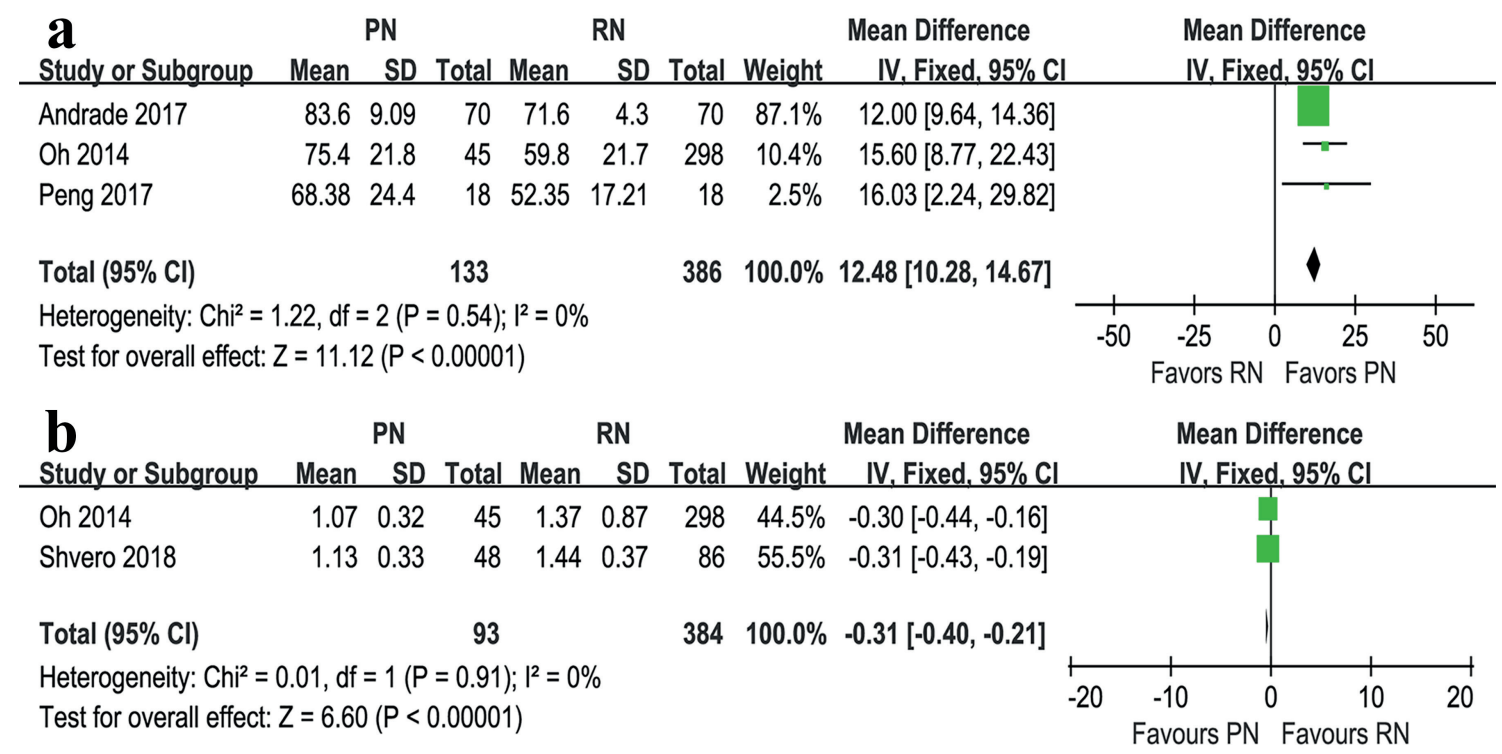

rative outcomes and postoperative renal function between PN and RN for treating pT3a RCC. We found significantly better serum creatinine and eGFR levels in the postoperative period among patients undergoing PN compared with the RN group. PN offered equivalent oncologic outcomes among patients with pT3a RCC. Moreover, there were no significant differences between the two groups with regard to surgical complications, EBL and operative time.

The impact of oncologic outcomes was an indispensable factor when choosing PN or RN. Our meta-analysis found no significant differences regarding oncologic outcomes. There were also no differences in recurrence and metastasis. Andrade et al. (18) reported no differences in recurrence $(2.9 \%$ vs. $1.4 \%, \mathrm{P}=1.00)$ and metastasis $(8.6 \%$ vs. $5.7 \%, \mathrm{P}=0.74)$. Similarly, using the Cox proportional hazard model, Shvero et al. (20) demonstrated that surgical type was not a predictive factor for recurrence $(\mathrm{P}=0.978)$ and metastatic progression $(\mathrm{P}=0.972)$. Recently, some studies have demonstrated that PN offers equivalent cancer control compared with RN in treating large RCC, and Shvero et al. (23) suggested that PN yielded similar oncologic outcomes for pT3a RCC at the 5-year follow-up. Moreover, Thompson et al. (24) showed that compared with RN, PN had equivalent CSS and OS for masses between 4 and $7 \mathrm{~cm}$. In addition to these studies, two German centers reported that CSS was similar between two groups for tumors $>7 \mathrm{~cm}$ (25). Furthermore, the experience of successful PN even for pT3b renal tumors confined to the renal vein has also been published by some centers $(26,27)$. Although these studies from single or multiple centers support PN, we sought to analyze the data of surgical complications and postoperative renal function. Moreover, patients with robust renal function might be more suitable for RN because no survival advantage was found, though a significant positive margin difference favored RN (Figure-6C).

Surgical complications are a significant factor to consider when choosing PN or RN. We report that no significant differences were found regarding estimated blood loss (EBL). Our results also showed a trend toward a shorter operative time in the PN group $(\mathrm{P}=0.06)$, but without a significant difference, which was unlikely to be clinically significant. We observed a lack of a sufficient number of studies reporting surgical complications; indeed, only one of the included studies (Oh 2014) reported no significant differences in intraoperative complications (15.6\% vs. $14.4 \%, \mathrm{P}=0.842$ ) and postoperative complications (13.3\% vs. $12.4 \%, \mathrm{P}=0.844$ ) among pT3a RCC pa- 
Table 2 - Subgroup analyses for overall survival, recurrence free survival and cancer specific survival.

\begin{tabular}{|c|c|c|c|c|c|c|c|c|c|c|c|c|}
\hline \multirow[b]{2}{*}{ Group } & \multicolumn{4}{|c|}{ OS } & \multicolumn{4}{|c|}{ RFS } & \multicolumn{4}{|c|}{ CSS } \\
\hline & $\begin{array}{l}\text { No.of } \\
\text { studies }\end{array}$ & $\mathrm{HR}(95 \% \mathrm{Cl})$ & $P$ & $\begin{array}{l}12 \\
(\%)\end{array}$ & $\begin{array}{l}\text { No.of } \\
\text { studies }\end{array}$ & $\mathrm{HR}(95 \% \mathrm{Cl})$ & $P$ & $\begin{array}{l}12 \\
(\%)\end{array}$ & $\begin{array}{l}\text { No.of } \\
\text { studies }\end{array}$ & $\mathrm{HR}(95 \% \mathrm{Cl})$ & $\mathrm{P}$ & $\begin{array}{l}12 \\
(\%)\end{array}$ \\
\hline Total & 4 & $0.92(0.26-3.30)$ & 0.89 & 0 & 8 & $1.26(0.70-2.29)$ & 0.44 & 0 & 5 & $1.01(0.64-1.58)$ & 0.98 & 0 \\
\hline \multicolumn{13}{|l|}{ Upstaging } \\
\hline Yes & 1 & $0.74(0.02-27.80)$ & 0.87 & NA & 4 & $1.47(0.71-3.06)$ & 0.30 & 0 & 1 & $0.89(0.02-33.55)$ & 0.95 & NA \\
\hline No & 3 & $0.95(0.24-3.71)$ & 0.94 & 0 & 4 & $0.91(0.32-2.55)$ & 0.86 & 0 & 4 & $1.00(0.64-1.58)$ & 0.99 & 0 \\
\hline \multicolumn{13}{|c|}{ Adjustment/matching } \\
\hline Yes & 1 & $0.89(0.15,5.41)$ & 0.9 & NA & 1 & $1.05(0.08,14.58)$ & 0.94 & NA & 3 & $1.01(0.64,1.61)$ & 0.95 & 0 \\
\hline No & 3 & $0.94(0.15,5.79)$ & 0.95 & 0 & 7 & $1.27(0.69,2.35)$ & 0.44 & 0 & 2 & $0.87(0.13,5.97)$ & 0.89 & 0 \\
\hline \multicolumn{13}{|l|}{ Study center } \\
\hline Single & 2 & $0.86(0.17-4.32)$ & 0.85 & 0 & 4 & $1.49(0.70-3.16)$ & 0.30 & 0 & 2 & $0.89(0.14-5.82)$ & 0.9 & 0 \\
\hline Multiple & 1 & $1.00(0.08-12.10)$ & 1.00 & NA & 3 & $0.88(0.31-2.51)$ & 0.81 & 0 & 3 & $1.01(0.63-1.61)$ & 0.97 & 0 \\
\hline NS & 1 & $1.07(0.02-51.87)$ & 0.97 & NA & 1 & $1.47(0.11-19.96)$ & 0.77 & NA & NA & NA & NA & NA \\
\hline \multicolumn{13}{|l|}{ Tumor size a } \\
\hline$\leq 4 \mathrm{~cm}$ & 1 & $0.93(0.72-1.20)$ & 0.56 & NA & NA & NA & NA & NA & 2 & $0.91(0.63-1.30)$ & 0.59 & 0 \\
\hline $4-7 \mathrm{~cm}$ & 2 & $0.89(0.65-1.22)$ & 0.48 & 0 & 3 & $1.56(0.69-3.54)$ & 0.29 & 0 & 4 & $0.90(0.58-1.40)$ & 0.65 & 0 \\
\hline $7-16 \mathrm{~cm}$ & 1 & $0.99(0.67-1.46)$ & 0.95 & NA & NA & NA & NA & NA & 1 & $1.07(0.66-1.75)$ & 0.77 & NA \\
\hline Mixed & 2 & $0.92(0.18-4.73)$ & 0.92 & 0 & 2 & $0.90(0.19-4.21)$ & 0.89 & 0 & NA & NA & NA & NA \\
\hline NS & 1 & $0.74(0.02-27.80)$ & 0.87 & NA & 3 & $1.03(0.36-2.93)$ & 0.96 & 0 & 1 & $0.89(0.02-33.55)$ & 0.95 & NA \\
\hline \multicolumn{13}{|c|}{ Follow-up time (m) } \\
\hline$\geq 50$ & 1 & $1.00(0.08-12.10)$ & 1.00 & NA & 2 & $0.97(0.24-3.92)$ & 0.96 & 0 & 2 & $1.19(0.27-5.14)$ & 0.82 & 0 \\
\hline$<50$ & 3 & $0.89(0.20-3.94)$ & 0.88 & 0 & 6 & $1.34(0.69-2.58)$ & 0.39 & 0 & 3 & $0.99(0.62-1.59)$ & 0.96 & 0 \\
\hline
\end{tabular}

$\mathbf{O S}=$ overall survival; $\mathbf{R F S}=$ recurrence free survival; $\mathbf{C S S}$ = cancer specific survival; $\mathbf{H R}=$ hazard ratio; $\mathbf{N A}=$ not available; $\mathbf{N S}=$ not specified

${ }^{a}$ one included study (Srivastava 2018) reported three separate subgroup analyses for the same data set ( $\leq 4 \mathrm{~cm}, 4-7 \mathrm{~cm}$ and $7-16 \mathrm{~cm}$ ).

tients (14). However, EORTC 30904 found that PN was associated with more complications than $\mathrm{RN}$, mostly hemorrhagic (28). In fact, the possible risk might be greater for more complicated and larger RCC, which requires a wider parenchyma resection, longer warm ischemia time and renal function reconstruction $(29,30)$. Therefore, our findings suggest that the potential advantages of PN need to offset the possibility of high surgical risk, especially for larger RCC.

The influence of kidney functional protection is essential when comparing PN and RN. Recently, some studies have demonstrated an association of RN with worse eGFR and a higher 
Figure S1 - Begg's and Egger's tests for comparisons of HR of OS (a), RFS (b) and CSS (c) associated with PN versus RN.
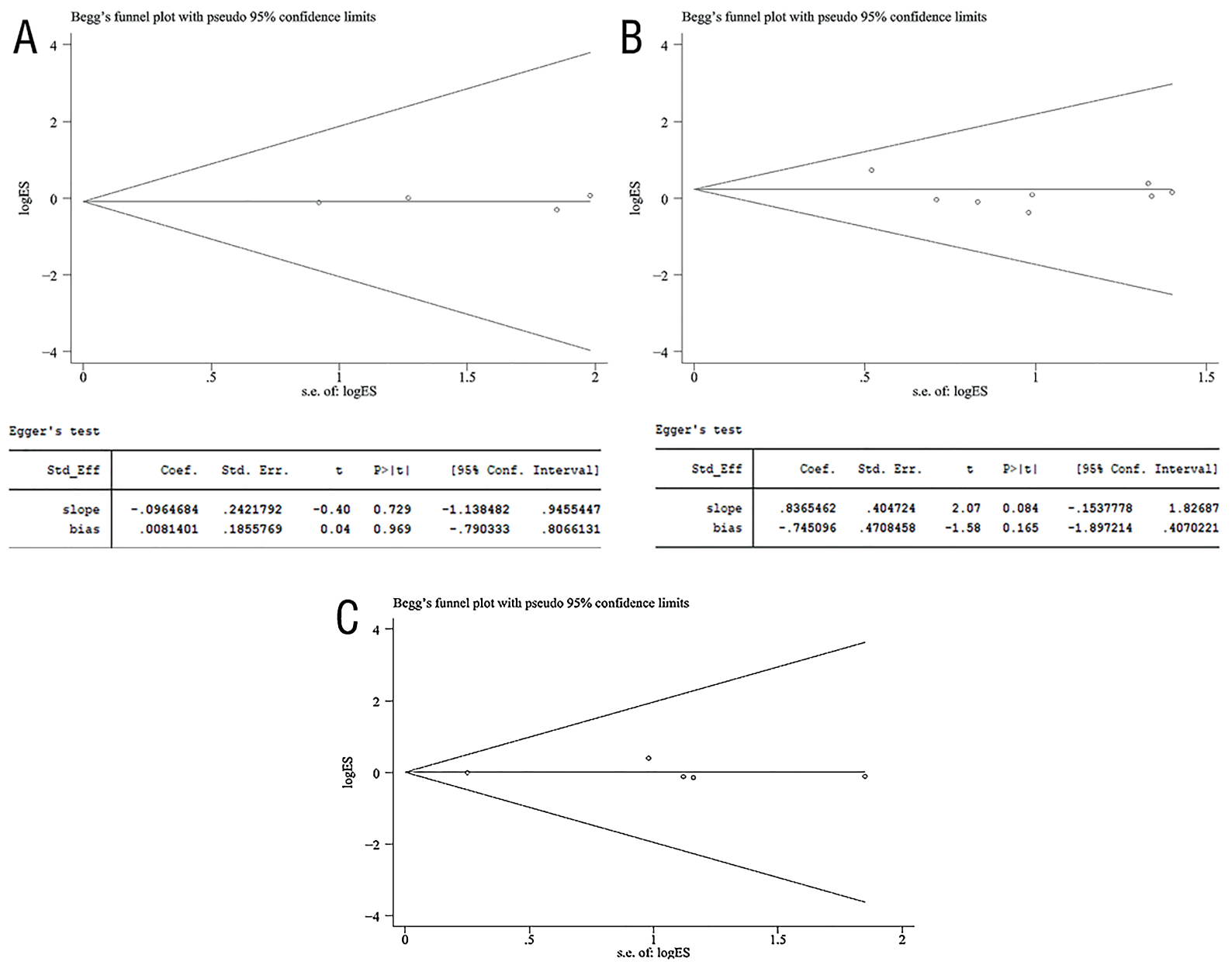

Egger's test

\begin{tabular}{r|rrrrrr}
\hline Std_Eff & Coef. & Std. Exr. & $\tau$ & $p>|\tau|$ & \multicolumn{2}{c}{ [S5s Conf. Interval] } \\
\hline slope & -.0032125 & .0891643 & -0.04 & 0.974 & -.2869729 & .280548 \\
bias & .023569 & .1732348 & 0.14 & 0.900 & -.5277414 & .5748795 \\
\hline
\end{tabular}

danger of cardiovascular events than PN $(6,31$, 32). Furthermore, worse renal function has been associated with all-cause mortality and some cardiovascular risk factors, including increased inflammatory factors, anemia, artery calcification, endothelial dysfunction, left ventricular hypertrophy and high levels of apolipoprotein (33). A study including 1331 patients showed that the risk of cardiovascular events after nephrectomy was significant and that PN could independently reduce the risk of cardiovascular events compared with RN after interpreting latent confounders and selection biases secondary to baseline cardiovascular risk Kim et al. (34). Additionally, in a systematic review and meta-analysis of 34 included articles, Lane, et al. (35) found a cumulative 61\% decrease in the risk of severe chronic kidney disease (CKD) and a 19\% risk decrease of all-cause mortality for patients undergoing PN. Although EORTC 30904 suggested that the favorable effect of PN on postoperative eGFR did not lead to improved OS with a median follow-up of 9.3 years $(3,28)$, patients undergoing PN would undoubtedly have higher survival quality. These findings 
may be explained by recent studies favoring the concept that CKD is not equivalent (35). According to recently published studies, patients have a strong annual reduction in renal function with preexisting CKD (CKD-M) compared to surgical CKD (CKD-S), close to 5\% versus 0.7\%. Additionally, Lane et al. (36) suggested higher rates of progressive reduction in kidney function, all-cause mortality and non-renal cancer mortality for CKD-M compared to CKD-S, whereas CKD-S had better survival, with no CKD for a median follow-up of 9.4 years. Moreover, they confirmed the significance of renal functional protection by demonstrating an association between baseline eGFR of $45 \mathrm{~mL} / \mathrm{min}$ and worse results after surgery (36).

Some limitations should be considered in our meta-analysis. First, our results might have been influenced by potential bias because retrospective studies and conference abstracts were excluded. Second, some included studies did not completely match some important factors, such as tumor size, which may have an impact on final outcomes. Third, we were unable to completely control for confounding factors (for example, surgical approach), which were unavailable in some articles, that can influence the final results. Fourth, some data (3-year all-cause mortality, 5-year all-cause mortality, 3-year recurrence rate, 5-year recurrence rate, 2-year CSS and 5-year CSS) were extracted from survival curves, which may have led to deviations from the real data. Fifth, the limited number of studies regarding surgical complications and perioperative outcomes might have resulted in unreliable estimates. Sixth, there was significant heterogeneity (65\%-81\%) for some comparisons (3-year recurrence rate and 5-year recurrence rate), which would weaken the reliability of these results.

\section{CONCLUSIONS}

Our meta-analysis suggests that PN may be more suitable for pT3a RCC, as it offers similar oncologic control and better renal functional preservation. Nevertheless, due to the inherent limitations of this meta-analysis, additional large-scale, high- -quality articles are required to better determine the role of PN in complicated clinical situations.

\section{ABBREVIATIONS}

RN = Radical Nephrectomy;

PN = Partial Nephrectomy;

RCC $=$ Renal cell carcinoma;

OS = Overall survival;

CSS = Cancer-specific survival;

RFS = Recurrence-free survival;

$\mathrm{EBL}=$ Estimated blood loss;

eGFR = Estimated glomerular filtration rate;

RCT $=$ Randomized control trial;

pT3a = Pathological T3a;

HR = Hazard ratio;

NOS = Newcastle-Ottawa Scale;

CIs $=$ Confidence intervals;

$\mathrm{RR}=$ Risk ratios;

WMD $=$ Weighted mean difference.

\section{ACKNOWLEDGEMENTS}

The authors thank professor Jichun Liu, MD (Department of Cardio-Thoracic Surgery, The second affiliated hospital of Nanchang University) for his statistical advice and professor Xiaoshu Cheng, MD, PhD (Department of Cardiology, The second affiliated hospital of Nanchang University) for his data collection.

\section{FUNDING INFORMATION}

National Natural Science Foundation of

China: 81560345

Natural Science Foundation of

Jiangxi Province: 20181BAB215027)

\section{CONFLICT OF INTEREST}

None declared.

\section{REFERENCES}

1. Siegel RL, Miller KD, Jemal A. Cancer statistics, 2018. CA Cancer J Clin. 2018;68:7-30.

2. Lane BR, Kattan MW. Prognostic models and algorithms in renal cell carcinoma. Urol Clin North Am. 2008;35:613-25. 
3. Scosyrev E, Messing EM, Sylvester R, Campbell S, Van Poppel $\mathrm{H}$. Renal function after nephron-sparing surgery versus radical nephrectomy: results from EORTC randomized trial 30904. Eur Urol. 2014;65:372-7.

4. Ljungberg B, Bensalah K, Canfield S, Dabestani S, Hofmann $\mathrm{F}$, Hora M, et al. EAU guidelines on renal cell carcinoma: 2014 update. Eur Urol. 2015;67:913-24.

5. Breau RH, Crispen PL, Jimenez RE, Lohse CM, Blute ML, Leibovich BC. Outcome of stage T2 or greater renal cell cancer treated with partial nephrectomy. J Urol. 2010;183:903-8.

6. Huang WC, Levey AS, Serio AM, Snyder M, Vickers AJ, Raj GV, et al. Chronic kidney disease after nephrectomy in patients with renal cortical tumours: a retrospective cohort study. Lancet Oncol. 2006;7:735-40.

7. Go AS, Chertow GM, Fan D, McCulloch CE, Hsu CY. Chronic kidney disease and the risks of death, cardiovascular events, and hospitalization. N Engl J Med. 2004;351:1296-305. Erratum in: N Engl J Med. 2008;18:4.

8. Parmar MK, Torri V, Stewart L. Extracting summary statistics to perform meta-analyses of the published literature for survival endpoints. Stat Med. 1998;17:2815-34. Erratum in: Stat Med. 2004;23:1817.

9. Tierney JF, Stewart LA, Ghersi D, Burdett S, Sydes MR. Practical methods for incorporating summary time-to-event data into meta-analysis. Trials. 2007;8:16.

10. Wells GA, Shea BJ, O'Connell D: The Newcastle-Ottawa Scale (NOS) for assessing the quality of non-randomized studies in meta-analysis. Appl Eng Agric. 2014; 18:727-34.

11. Jeldres C, Patard JJ, Capitanio U, Perrotte P, Suardi N, Crepel $\mathrm{M}$, et al. Partial versus radical nephrectomy in patients with adverse clinical or pathologic characteristics. Urology. 2009;73:1300-5.

12. Hansen J, Sun M, Bianchi M, Rink M, Tian Z, Hanna N, et al. Assessment of cancer control outcomes in patients with high-risk renal cell carcinoma treated with partial nephrectomy. Urology. 2012;80:347-53.

13. Polo G, Crouzet S, Poissonnier L, Roux A, Deculier E, Martin $X$, et al. [Partial versus radical nephrectomy for renal cell carcinoma involving peri renal fat. Oncological and functional outcomes]. Prog Urol. 2012;22:388-96.

14. Oh JJ, Byun SS, Lee SE, Hong SK, Lee ES, Kim HH, et al. Partial nephrectomy versus radical nephrectomy for nonmetastatic pathological T3a renal cell carcinoma: a multiinstitutional comparative analysis. Int J Urol. 2014;21:352-7.

15. Jeong SH, Kim JK, Park J, Jeon HJ, Yoon MY, Jeong CW, et al. Pathological T3a Upstaging of Clinical T1 Renal Cell Carcinoma: Outcomes According to Surgical Technique and Predictors of Upstaging. PLoS One. 2016;11:e0166183.
16. Nayak JG, Patel P, Saarela O, Liu Z, Kapoor A, Finelli A, et al. Pathological Upstaging of Clinical T1 to Pathological T3a Renal Cell Carcinoma: A Multi-institutional Analysis of Shortterm Outcomes. Urology. 2016;94:154-60.

17. Shah PH, Moreira DM, Patel VR, Gaunay G, George AK, Alom $\mathrm{M}$, et al. Partial Nephrectomy is Associated with Higher Risk of Relapse Compared with Radical Nephrectomy for Clinical Stage T1 Renal Cell Carcinoma Pathologically Up Staged to T3a. J Urol. 2017;198:289-96.

18. Andrade HS, Zargar H, Akca O, Kara O, Caputo PA, Ramirez D, et al. Is Robotic Partial Nephrectomy Safe for T3a Renal Cell Carcinoma? Experience of a High-Volume Center. J Endourol. 2017;31:153-7.

19. Peng D, He ZS, Li XS, Tang Q, Zhang L, Yang KW, et al. Partial nephrectomy for T3aNOMO renal cell carcinoma: shall we step forward? Int Braz J Urol. 2017;43:849-56.

20. Shvero A, Nativ O, Abu-Ghanem Y, Zilberman D, Zaher B, Levitt M, et al. Oncologic Outcomes of Partial Nephrectomy for Stage T3a Renal Cell Cancer. Clin Genitourin Cancer. 2018;16:e613-e617.

21. Srivastava A, Patel HD, Joice GA, Semerjian A, Gorin MA, Johnson MH, et al. Incidence of T3a up-staging and survival after partial nephrectomy: Size-stratified rates and implications for prognosis. Urol Oncol. 2018;36:12.e7-12. e13.

22. Lee H, Lee M, Lee SE, Byun SS, Kim HH, Kwak C, et al. Outcomes of pathologic stage T3a renal cell carcinoma up-staged from small renal tumor: emphasis on partial nephrectomy. BMC Cancer. 2018;18:427.

23. Shvero A, Zilberman D, Mor Y, Kaver I, Fridman E, Portnoy 0 , et al. Oncologic outcome after partial nephrectomy compared with radical nephrectomy for primary renal tumors in stage T3a. J Urol. 2016, 195:e27.

24. Thompson RH, Siddiqui S, Lohse CM, Leibovich BC, Russo $P$, Blute ML. Partial versus radical nephrectomy for 4 to $7 \mathrm{~cm}$ renal cortical tumors. J Urol. 2009;182:2601-6.

25. Becker F, Roos FC, Janssen M, Brenner W, Hampel C, Siemer $S$, et al. Short-term functional and oncologic outcomes of nephron-sparing surgery for renal tumours $\geq 7 \mathrm{~cm}$. Eur Urol. 2011;59:931-7.

26. Kolla SB, Ercole C, Spiess PE, Pow-Sang JM, Sexton WJ. Nephron-sparing surgery for pathological stage T3b renal cell carcinoma confined to the renal vein. BJU Int. 2010;106:1494-8.

27. Woldu SL, Barlow LJ, Patel T, Hruby GW, Benson MC, McKiernan JM. Single institutional experience with nephronsparing surgery for pathologic stage T3bNxMO renal cell carcinoma confined to the renal vein. Urology. 2010;76:639-42. 
28. Van Poppel H, Da Pozzo L, Albrecht W, Matveev V, Bono A, Borkowski $A$, et al. A prospective randomized EORTC intergroup phase 3 study comparing the complications of elective nephron-sparing surgery and radical nephrectomy for low-stage renal cell carcinoma. Eur Urol. 2007;51:160615.

29. Van Poppel H, Joniau S, Albersen M. Nephron Sparing for Renal Cell Carcinoma: Whenever Possible? Eur Urol Focus. 2016;2:656-9.

30. Mir MC, Derweesh I, Porpiglia F, Zargar H, Mottrie A, Autorino R. Partial Nephrectomy Versus Radical Nephrectomy for Clinical T1b and T2 Renal Tumors: A Systematic Review and Meta-analysis of Comparative Studies. Eur Urol. 2017;71:606-17.

31. Scosyrev E, Messing E, Campbell S. Radical versus partial nephrectomy for a small renal mass: does saving nephrons save lives? Expert Rev Anticancer Ther. 2013;13:1349-51.

32. Yap SA, Finelli A, Urbach DR, Tomlinson GA, Alibhai SM. Partial nephrectomy for the treatment of renal cell carcinoma (RCC) and the risk of end-stage renal disease (ESRD). BJU Int. 2015;115:897-906.

33. Capitanio U, Terrone C, Antonelli A, Minervini A, Volpe A, Furlan $\mathrm{M}$, et al. Nephron-sparing techniques independently decrease the risk of cardiovascular events relative to radical nephrectomy in patients with a $\mathrm{T} 1 \mathrm{a}-\mathrm{T} 1 \mathrm{~b}$ renal mass and normal preoperative renal function. Eur Urol. 2015;67:683-9.

34. Kim SP, Murad MH, Thompson RH, Boorjian SA, Weight CJ, Han LC, et al. Comparative Effectiveness for Survival and Renal Function of Partial and Radical Nephrectomy for Localized Renal Tumors: A Systematic Review and MetaAnalysis. J Urol. 2012;18. [Epub ahead of print]

35. Lane BR, Demirjian S, Derweesh IH, Riedinger CB, Fergany $\mathrm{AF}$, Campbell SC. Is all chronic kidney disease created equal? Curr Opin Urol. 2014;24:127-34.

36. Lane BR, Demirjian S, Derweesh IH, Takagi T, Zhang Z, Velet L, et al. Survival and Functional Stability in Chronic Kidney Disease Due to Surgical Removal of Nephrons: Importance of the New Baseline Glomerular Filtration Rate. Eur Urol. 2015;68:996-1003.

Correspondence address: Wenxiong Zhang, MD Department of Thoracic Surgery

The second affiliated hospital of Nanchang University No. 1, Minde Rd, Nanchang, P. R. China 330006 E-mail: zwx123dr@126.com 